\title{
Teaching Recurrent Laryngeal Nerve Dissection to Residents in Head and Neck Endocrine Surgery
}

\author{
Krishnamurthi Sundaram, Behrad Ben Aynehchi
}

\begin{abstract}
Background: Various approaches can be employed when teaching thyroidectomy. Delivery of the gland prior to complete dissection and identification of the recurrent laryngeal nerve $(R L N)$ is sometimes utilized in the absence of laryngeal nerve monitoring (LNM), while identification and dissection of the RLN from the gland is more feasible in the presence of LNM. We hypothesized that teaching RLN dissection technique to residents as primary surgeons (using loupes and nerve monitoring) did not increase postoperative morbidity in head and neck endocrine surgery.
\end{abstract}

Materials and methods: In 101 consecutive patients who underwent thyroidectomies/parathyroidectomies, we have modified our technique to teach our residents consistent early identification of the RLN in the paratracheal region by direct visualization (loupe magnification) and nerve stimulation. All patients had preoperative fiberoptic laryngoscopy and a repeat laryngoscopy 1 week after surgery. Presence or absence of true vocal fold paralysis/paresis was documented.

Results: One hundred and nineteen surgical procedures were available for study. One patient developed a permanent vocal fold paralysis ( $1 / 119$ or $0.84 \%)$. There was another patient with a vocal fold paresis which recovered in 4 weeks $(1 / 119$ or $0.84 \%)$.

Conclusion: The RLN dissection technique with early identification, dissection and preservation of the nerve using magnification combined with nerve monitoring has emerged as an important resident teaching tool. The morbidity rate is acceptable. Notably in light of the increasing rates of thyroid surgery and LNM utilization, resident physicians may benefit from exposure to this technique as a component of their training.

Keywords: Teaching, Recurrent laryngeal nerve, Dissection, Nerve monitoring, Magnification.

How to cite this article: Sundaram K, Aynehchi BB. Teaching Recurrent Laryngeal Nerve Dissection to Residents in Head and Neck Endocrine Surgery. Int J Head and Neck Surg 2012;3(1):5-7.

\section{Source of support: Nil}

Conflict of interest: None declared

\section{INTRODUCTION}

In a recent survey of the members of the American Head and Neck Society, we found that the percentage of members using laryngeal nerve monitoring, at least in some cases, has gradually increased to $65 \%$ of those surveyed (unpublished data). This is in contrast to a survey of the members of the American Association of Endocrine Surgeons and registrants of their 2006 annual meeting where only $37.1 \%$ of the respondents used neuromonitoring. ${ }^{1}$ Another study where a questionnaire was mailed to 1685 randomly selected otolaryngologists had 685 questionnaires returned. Of the respondents $44.9 \%$ used a monitor in their current practice. ${ }^{2}$ But many studies by experienced endocrine surgeons show no significant difference in rates of temporary paresis or permanent paralysis of the recurrent laryngeal nerve during thyroidectomy with or without laryngeal nerve monitoring. ${ }^{3}$ Careful review of the technique used by these experienced authors shows no major difference in technique on the monitored or nonmonitored side except stimulation to confirm identification of the nerve at the beginning and stimulation at the end of the dissection to confirm a functioning nerve. ${ }^{4}$

Various approaches can be employed in thyroidectomy. Delivery of the gland prior to complete dissection and identification of the recurrent laryngeal nerve (RLN) is sometimes utilized, while initial identification and dissection of the RLN from the gland seems more feasible in the presence of laryngeal nerve monitoring (LNM). In training residents as primary surgeons we have modified our technique to consistently perform early identification and dissection of the RLN in the paratracheal region by a combination of visual identification (loupe magnification) and nerve stimulation.

We hypothesized that teaching RLN dissection technique to residents as primary surgeons (using loupes and nerve monitoring) did not increase postoperative morbidity in head and neck endocrine surgery.

\section{MATERIALS AND METHODS}

A chart review of 101 consecutive cases of head and neck endocrine surgery using this technique was performed. There were 121 nerves at risk. IRB approval was obtained.

In our technique, the superior pole of the thyroid is exposed by dividing the sternothyroid muscle. Loupe magnification, nerve monitoring and the harmonic scalpel are used. The external branch of the superior laryngeal nerve (EBSLN) is identified and function confirmed using the nerve monitor. The superior pedicle vessels are taken individually and proximal ends ligated. The middle thyroid vein/veins are then divided. The thyroid lobe is then rotated medially. The superior and inferior parathyroid glands are identified and protected. The RLN is then identified and dissected out in the paratracheal region. The thyroid lobe is 
then dissected away from the nerve, as in a parotidectomy, using magnification and nerve monitoring. This technique is similar to the one described by Randolph et al. ${ }^{5}$ The RLN and its branches are dissected and preserved. The integrity of the motor division is confirmed with Prass probe stimulation. Even with large substernal goiters, the RLN is completely dissected away and protected before delivering the substernal component with blunt dissection. All patients had preoperative fiberoptic laryngoscopy and a repeat laryngoscopy 1 week after surgery. Presence or absence of true vocal fold paralysis/paresis was documented.

\section{RESULTS}

One hundred and nineteen surgical procedures in 101 patients were available for study. Two patients were excluded (one with preoperative vocal fold paralysis and one with ventilator-dependent respiratory failure). Patients' age ranged from 26 to 80 years. There were 22 males (22\%) and 79 females (78\%). There were 18 Caucasian patients (18\%), 58 African-Americans (57\%), 21 Hispanics (21\%) and 4 Asians (4\%). There were 20 total thyroidectomies: 15 for malignancy and five for benign disease; six substernal goiters, one revision thyroidectomy and 10 parathyroidectomies. One patient developed a permanent vocal fold paralysis $(1 / 119$ or $0.84 \%)$. The nerve section was discovered as it was being dissected off a substernal goiter. It was a ventrally placed nerve which did not respond to stimulation. It was immediately repaired using the ansa cervicalis. Patient's postoperative voice and swallowing function was acceptable and she did not require further surgical procedures. There was one patient with an unexplained vocal fold paresis which recovered in 4 weeks $(1 / 119$ or $0.84 \%)$.

\section{DISCUSSION}

There is anatomic variability in the presentation of the EBSLN as documented by Cernea. ${ }^{6}$ There are multiple

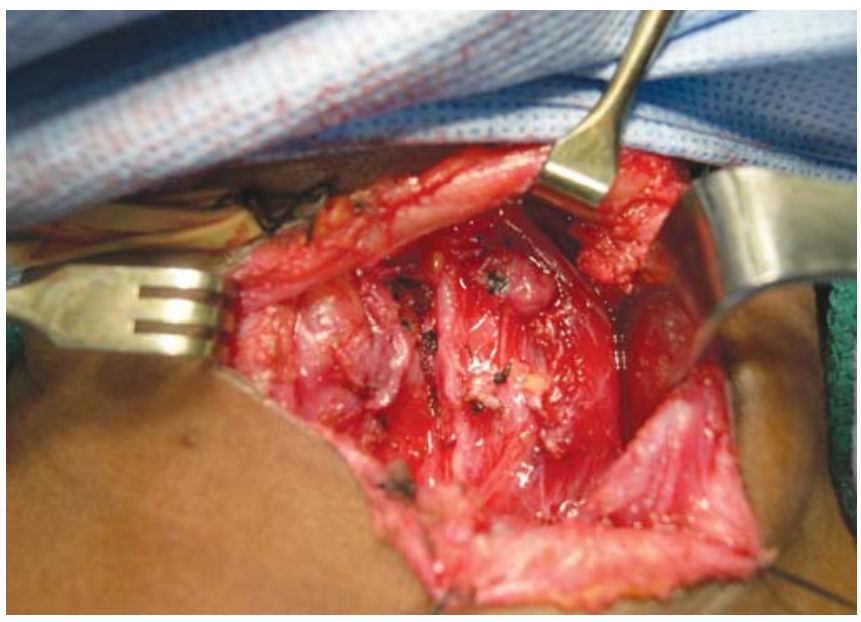

Fig. 1: Recurrent laryngeal nerve as a single trunk variations of the RLN as described by Randolph ${ }^{7}$ (Figs 1 and 2). In a cadaver dissection, Yalcin et al have described branching in $85.4 \%$ of the RLN. ${ }^{8}$ Usually, there are two divisions but on occasion there can be three divisions. These divisions can further subdivide before entering the larynx. The anterior division is considered the motor division but in some cases it is the posterior division that responds to stimulation. Because of these variations which cannot be predicted preoperatively, early identification of the RLN using magnification and nerve monitoring gives us the best chances of obtaining an optimum outcome. As the nerve is identified in the paratracheal region and the various divisions traced, the integrity of the motor division is confirmed by stimulation. To ensure protection of the nerve, the thyroid or the parathyroid gland is dissected away from the nerve before the gland is mobilized. Injury to the nerve can be immediately identified and repaired as was the case in one patient in our series where the ansa cervicalis was anastomosed to the RLN. In our recent systematic review of laryngeal reinnervation techniques, we did not find the superiority of any one technique. The specific method used is dictated by anatomic limitations. ${ }^{9}$

Intraoperative RLN monitoring is not considered the standard of care. It has its pitfalls and has a learning curve. ${ }^{10}$ Improper positioning of the electromyographic endotracheal tube (EMG-ET) can give rise to complications. ${ }^{11}$ When routine use of the EMG-ET is part of their training, residents quickly become familiar with these issues. Combining early identification, magnification and nerve monitoring, the RLN dissection technique is emerging as an important teaching tool in head and neck endocrine surgery. The incidence of temporary paresis was acceptable $(0.84 \%)$; the range reported is 0.4 to $7.2 \%{ }^{12}$ The incidence of permanent paralysis in our series was $0.84 \%$. The reported range is from 0 to $5.2 \%$. Dralle has calculated that a randomized controlled trial will need at least 40,000 patients per arm

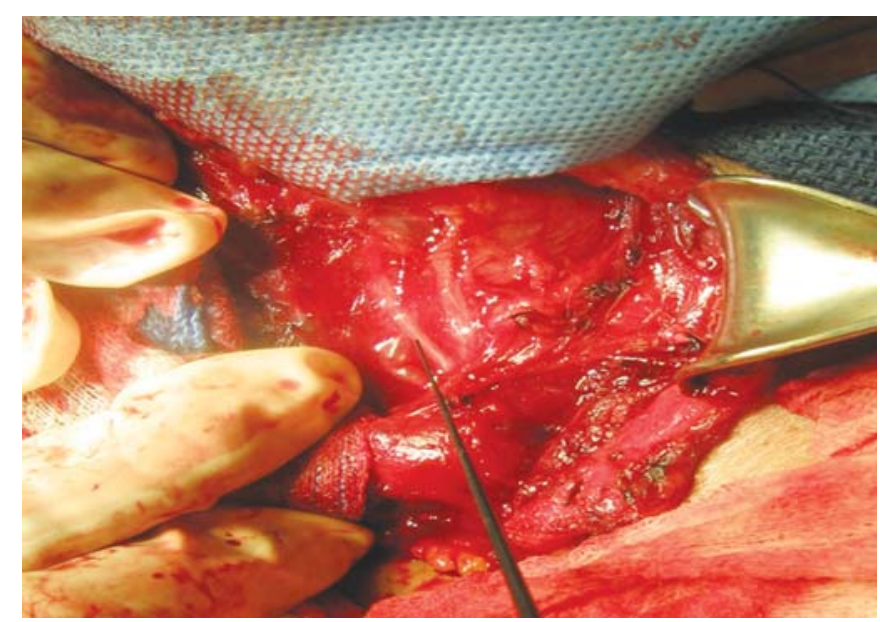

Fig. 2: Branching recurrent laryngeal nerve 
for adequate statistical power and may not be feasible to study these techniques. ${ }^{5}$

\section{CONCLUSION}

The RLN dissection technique with early identification, dissection and preservation of the nerve using magnification combined with nerve monitoring has emerged as an important resident teaching tool. There is no increase in morbidity. Notably in light of the increasing rates of thyroid surgery and LNM utilization, resident physicians may benefit from exposure to this technique as a component of their training. Prospective studies looking at voice and swallowing outcomes with this technique are necessary.

\section{REFERENCES}

1. Sturgeon C, Sturgeon T, Angelos P. Neuromonitoring in thyroid surgery: Attitudes, usage patterns and predictors of use among endocrine surgeons. World J Surg 2009;33:417-25.

2. Horne SK, Gal TJ, Brennan JA. Prevalence and patterns of intraoperative nerve monitoring for thyroidectomy. Otol Head Neck Surg 2007;136:952-56.

3. Higgins TS, Gupta R, Ketcham AS, Sataloff RT, Wadsworth $\mathrm{JT}$, et al. Recurrent laryngeal nerve monitoring versus identification alone on post-thyroidectomy true vocal fold palsy: A meta-analysis. Laryngoscope 2011;121:1009-17.

4. Shindo M, Chheda NN. Incidence of vocal cord paralysis with and without recurrent laryngeal nerve monitoring during thyroidectomy. Arch Otolaryngol Head Neck Surg 2007; 133:481-85.

5. Randolph GW, Dralle H, Abdullah H, Barczynski M, et al. With the international intraoperative monitoring study group, electrophysiologic recurrent laryngeal nerve monitoring during thyroid and parathyroid surgery: International standards guideline statement. Laryngoscope 2011;121:S1-16.

6. Cernea CR, Ferraz AR, Nishio S, Dutra A Jr, Hojaij FC, et al. Surgical anatomy of the external branch of the superior laryngeal nerve. Head Neck 1992;14(5):380-83.

7. Randolph GW. Surgical anatomy of the recurrent laryngeal nerve. Randolph GW (Ed). Surgery of the thyroid and parathyroid glands 2003 Elsevier Science (USA). Chapter 25, 300-42.

8. Yalcin B, Tugcu H, Canturk N, Ozan H. Laryngeal branching pattern of the inferior laryngeal nerve, before entering the larynx. Surg Radiol Anat 2006;28:339-42.

9. Aynehchi BB, McCoul ED, Sundaram K. Systematic review of laryngeal reinnervation techniques. Otol Head Neck Surg 2010;143:749-59.

10. Snyder SK, Hendricks JC. Intraoperative neurophysiology testing of the recurrent laryngeal nerve: Plaudits and pitfalls. Surgery 2005;138:1183-92.

11. Sundaram K. Prevention of main-stem bronchus intubation with the EMG endotracheal tube. Letters to the editor. Otol Head Neck Surg 2010;142:152.

12. Robertson ML, Steward DL, Gluckman JL, Welge J. Continuous laryngeal nerve integrity monitoring during thyroidectomy: Does it reduce risk of injury? Otol Head Neck Surg 2004;596-600.

\section{ABOUT THE AUTHORS}

\section{Krishnamurthi Sundaram}

Department of Otolaryngology, Head and Neck Surgery, State University of New York Downstate Medical Center, New York, USA

\section{Behrad Ben Aynehchi (Corresponding Author)}

Department of Otolaryngology, Head and Neck Surgery, State University of New York Downstate Medical Center, New York, USA e-mail: baynehchi@gmail.com 